\title{
A Hybrid Automated Intelligent COVID-19 Classification System Based on Neutrosophic Logic and Machine Learning Techniques Using Chest X-Ray Images
}

\author{
Ibrahim Yasser, Aya A. Abd El-Khalek, Abeer Twakol, \\ Mohy-Eldin Abo-Elsoud, Ahmed A. Salama, and Fahmi Khalifa
}

\begin{abstract}
To facilitate timely treatment and management of COVID-ap patients, efficient and quick identification of COVID-19 patients is of immense importance during the COVID-19 crisis. Technological developments in machine learning (ML) methods, edge computing, computer-aided medical diagnostic been utilized for COVID-19 Classification. This is mainly because of their ability to deal with Big data and their inherent robustness and ability to provide distinct output characteristics attributed to the underlying application. The contrary transcriptionpolymerase chain reaction is currently the clinical typical for COVID-19 diagnosis. Besides being expensive, it has low sensitivity and requires expert medical personnel. Compared with RT-PCR, chest X-rays are easily accessible with highly available annotated datasets and can be utilized as an ascendant alternative in COVID-19 diagnosis. Using X-rays, ML methods can be employed to identify COVID-19 patients by quantitively examining chest X-rays effectively. Therefore, we introduce an alternative, robust, and intelligent diagnostic tool for automatically
\end{abstract}

\section{Yasser $(\square)$}

Engineering Faculty, University of Mansoura, Mansoura 35516, Egypt

e-mail: Ibrahim_yasser@mans.edu.eg

A. A. Abd El-Khalek

Communications and Electronics Engineering Department, Nile Higher Institute for Engineering and Technology, Mansoura 35524, Egypt

A. Twakol

Computer Engineering Department, Engineering Faculty, Benha University,

Banha 13518, Egypt

M.-E. Abo-Elsoud · F. Khalifa

Electronics and Communications Engineering Department, Engineering Faculty, University of Mansoura, Mansoura 35516, Egypt

e-mail: fahmikhalifa@mans.edu.eg

\section{A. A. Salama}

Mathematics and Computer Science Department, Faculty of Sciences,

University of Port Said, Port Said 42522, Egypt

(C) The Author(s), under exclusive license to Springer Nature Switzerland AG 2022 
detecting COVID-19 utilizing available resources from digital chest X-rays. Our technique is a hybrid framework that is based on the fusion of two techniques, Neutrosophic techniques (NTs) and ML. Classification features are extracted from $\mathrm{X}$-ray images using morphological features (MFs) and principal component analysis (PCA). The ML networks were trained to classify the chest X-rays into two classes: positive (+ve) COVID-19 patients or normal subjects (or -ve). The experimental results are performed based on a sample from a collected comprehensive image dataset from several hospitals worldwide. The classification accuracy, precision, sensitivity, specificity and F1-score for the proposed scheme was $98.46 \%, 98.19 \%, 98.18 \%, 98.67 \%$, and $98.17 \%$. The experimental results also documented the high accuracy of the proposed pipeline compared to other literature techniques.

Keywords COVID-19 - Artificial intelligence - Machine learning • Neutrosophic techniques - Computer-aided diagnostic tool

\section{Introduction}

Coronavirus Disease-2019 (COVID-19) caused by the so-called severe acute respiratory syndrome coronavirus2 (or SARS-CoV-2) is a devastating worldwide epidemic. In March 2020, the world health organization (WHO) announced that SARS-CoV-2 (or COVID-19) had become an unprecedented public health crisis. SARS-CoV-2 is known for its high transmutability and pathogenicity. Many research laboratories worldwide are working on developing a clinical antiviral drug and/or vaccine to help control the spread of the virus. Governments in various countries have implemented flight restrictions and limits on the border crossing. Also, public awareness of hygiene and social distancing has been imposed. However, the virus continues to unfold at a rapid pace. While the general population encountered mild to severe respiratory disease with COVID-19, others have had fatal pneumonia [1]. Several analysis within medicine, clinical, and computing are failing to organize proactive plans of action for COVID-19 with established research goals. As this illness is very infectious, the foremost fascinating interference technique is to spot infected patients to contain the spread; therefore, the transmission chain is interrupted [2]. Medical screening tool that is currently in use for COVID-19 diagnosis is RT-PCR, which stands for reverse transcription enzyme chain reaction. RT-PCR is complex manually employed, and it is also time consuming. Furthermore, it only has a rate of positivity $63 \%$. Additionally, the swab take a look at involves separation for the test protocol, whereas chest X-ray detection is simply managed [3]. In addition, there's a severe lack of supplies, that results in an interruption in tries to eradicate disease [4]. Delays in take a look at results will result in infected patients communication with and infecting healthy patients within the process. The other diagnostic approaches of COVID-19 embrace the study of medical specialty signs, epidemiologic histories and positive 
pradiographic images [computed tomography (CT)/chest radiography (CXR)] also as positive morbific checks. The majority of COVID-19 cases have similar decisions for X-ray imaging, together with longitudinal, multifocal, ground-glass natural process with peripheral or point distribution, chiefly within the lower lobes of the earlying process and late stage respiratory organ consolidation [5]. Although CT chest may be a lot of economical imaging procedure for lung-related dis-ease diagnosis, CXR is favored as a result of its pronto quicker, accessible and cheaper than with CT. Since infection with COVID-19 attacks epithelial tissue our metastasis tract, X-ray images is accustomed diagnose respiratory organ pneumonia, lung inflammation, abscesses, Associate in nursing/or swollen bodily fluid nodes. The studies advocate the necessity for a computerised classification for speedy diagnosis. Not only manual interpretations of X-rays is time overwhelming, it also unable to satisfy the perform because of the restricted offer of X-ray radiologists or machine operators [6].

Classification of biomedical images is an emerged area of study to form the care sector additional promising. Amid the aftermath of the epidemic of COVID-19, many recent international studies round the world have adopted subtle ML algorithms and AI based mostly technologies to higher perceive the pattern of infective agent transmission, more improve diagnostic speed and accuracy. Not only that, but it would also support health care staff by reducing their interaction with COVID-19 patients, also. Furthermore, ML/AI-based system help to develop new, successful treatment interventions and theoretically classify the most vulnerable people. ML/ AI-based techniques are expected and to improve the accuracy forecast for both infectious and non-infectious disease screening and identification COVID-19 on chest X-ray scans. In the medical sector, AI is not used to eliminate human experiences, but to provide decision-making guidance for physicians in what they model. Figure 1 depicts how AI and ML techniques can be used in the fight against Covid-19.

The rest of this chapter is structured as follows. Section 2 shall precede the work involved and shall discuss the nature of the work given. Section 3 introduces the suggested solution to the characterization of COVID-19 and its stages using chest X-rays. The experimental findings shall be addressed in Sect. 4. Finally, the conclusion are laid out in Sect. 5.

\section{Related Work}

At present, in progress tries are created to develop novel diagnostic strategies victimization ML algorithms. During this regard, studies demonstrate the potential of AI and ML tools by proposing new models for speedy and valid SARS-CoV-2 diagnostic methods, such as: Apostolopoulos and Mpesiana [7] in their research, advanced convolutionary neural network structures were used to identify CXR photos. Transfer learning has been introduced to cope with a number of gift anomalies within the dataset. 2 datasets from slightly separate repositories are used 


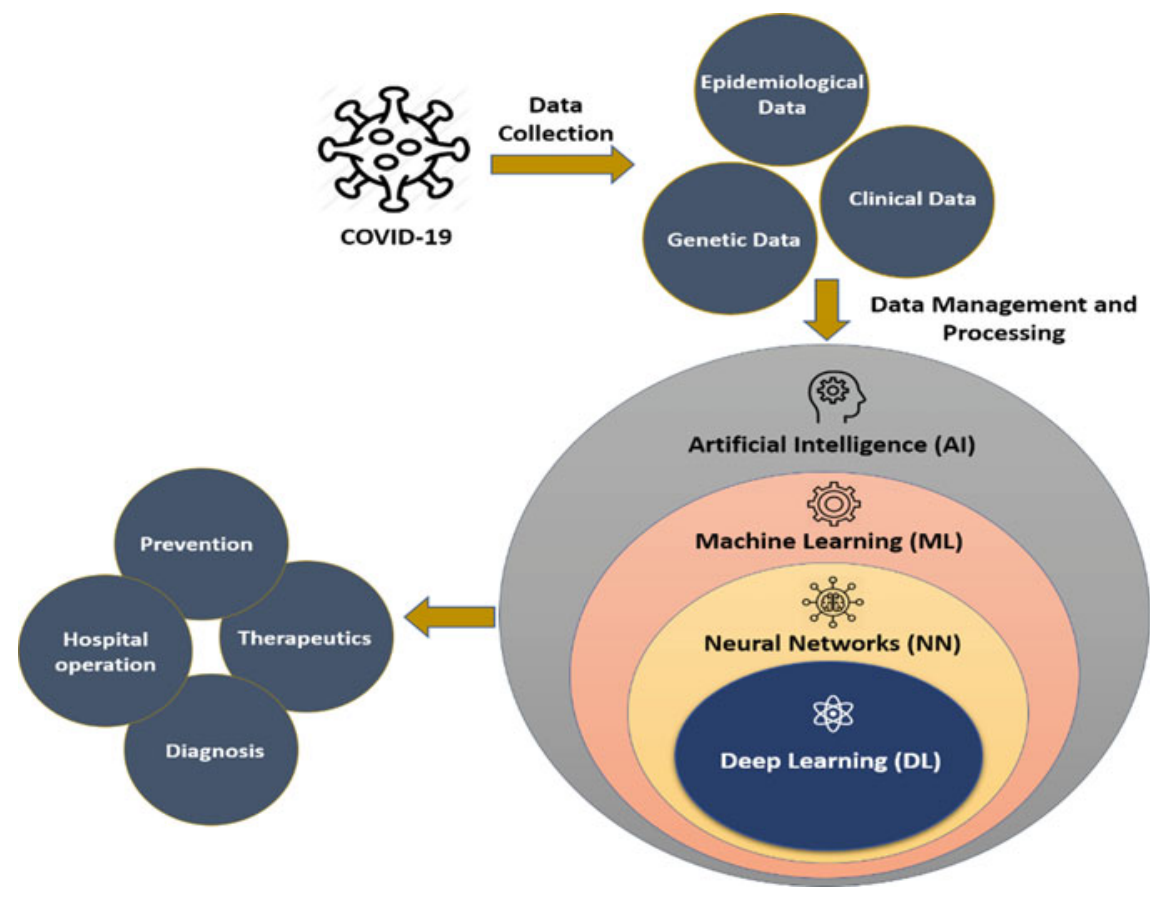

Fig. 1 Schematic illustration of artificial intelligence (AI) and machine learning (ML) techniques applied in the fight against covid-19

to research photographs in two classes: COVID-19 and the traditional state with a 97\% accuracy (sensitivity of $98.66 \%$ and specificity of $96.46 \%$ ). Sethy and Behera [8] proposed a CXR-b intelligent system for COVID-19 identification. Deep aspects of the CXRs have been identified and vector support machine (SVM) classifier was used. The study concluded that ResNet50 combined with SVM achieved best performances (accuracy, F1 score, Matthew's correlation coefficient (MCC) and Kappa were 95.38\%, 95.52\%, 91.41\% and 90.76\%, respectively). Another research by Khalifa et al. used generative adversarial networks (GAN) [9] to detect the respiratory disease from CXRs. They addressed the topic of over-processing and proclaimed their power by generating additional GAN images. The data collection contained 5863 CXR images for both natural and nuclear purposes. Well-known deep learning structures (e.g., GoogLeNet, AlexNet, Squeznet and ResNet) were used to diagnose pneumonia. Their work highlighted the fact that ResNet18 along with GAN outperformed other deep transport models. Afshar et al. [10] suggested a capsule network called COVID-CAPS instead of a conventional CNN network to communicate with a data set. COVID-CAPS are optimized to obtain an accuracy of $95.7 \%$, sensitivity of $90 \%$, and specificity of $95.8 \%$.

Wang et al. [11] provided a COVID-Net for COVID-19 cases detection from $\sim 14,000$ CXRs, with a precision of $83.5 \%$. Abbas et al. [12] rendered a pipeline 
using the pre-trained modified CNN architecture (DeTraC Decompose, Transfer, and Compose) for COVID-19 detection. They tested their approach on a database of 105 COVID-19, 80 normal subjects and 11 SARS X-rays. The method attained an accuracy of $95.12 \%$, sensitivity of $97.91 \%$, while the specificity was $91.87 \%$.

A systematic study concluded that CXR images throughout the chest can be used for the early diagnosis of COVID-19-infected patients. The literature review also documented the role of modern $\mathrm{AI} / \mathrm{ML}$ technology to solve the challenges during the outburst. Therefore, statistical models are used in this chapter to distinguish COVID-19 patients from CXR images in the chest; Therefor, the present work proposes a new automated intelligent ML algorithm combined with Neutrosophic techniques (NTs) to detect and classify COVID-19 pneumonia cases using X-ray images. The main objective is to reduce the error in detection while obtaining a more accurate diagnosis. The contributions are summarized as follows:

(i) Building a novel ML model in automatically and efficiently assist in early diagnosis of patients with COVID-19;

(ii) Achieving an empirical analysis of the proposed ML image classifier with NTs in the task of classifying COVID-19 disease using conventional chest $\mathrm{X}$-rays with lower cost than other imaging modalities like CT;

(iii) Comparing our technique with different models to document the robustness and the most accurate results of our method using the available X-rays;

(iv) Our analysis pipeline supports multidisciplinary researchers in developing advanced AI and ML techniques that can help fight the outbreak of COVID-19.

\section{The Proposed COVID-19 Diagnostic System}

The proposed architecture to accomplish the diagnostic procedure and for detecting the existence of COVID-19 using X-ray images as seen in Fig. 2, consisting of four phases, (i) preprocessing step; (ii) Feature extraction phase; (iii) operation of the NT process; and (iv) classification phase of the ML. These steps are listed in detail in the following paragraphs.

\subsection{Pre-processing}

The CXR images within the collective knowledge set accommodates uncalled-for objects resembling bright text, icons, differing resolutions, varied shapes, textures and morphological characteristics, usually of various colours, proportions, positioning, component level noise so on, that necessitate its pre-processing. Various pre-processing techniques are employed in this work to supply COVID-19 coaching files. Every image is regenerate as follows: (i) noise extracted to eliminate 


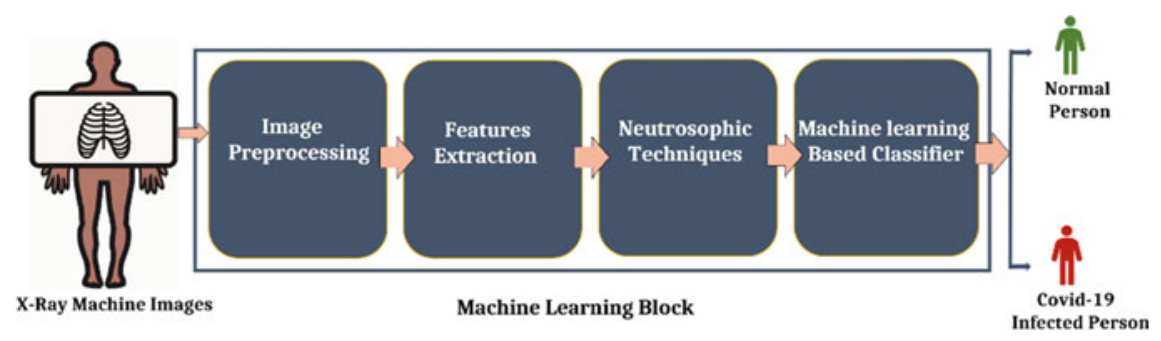

Fig. 2 The proposed block diagram for the COVID-19 classification pipeline

constituent level noise for many ac-curate images, so illuminated textual and semantic noise are curbed by a photomask generated using binary thresholds, as indicated by Eq. 1 [13], (ii) turned bound images that aren't utterly set in a very horizontal position. The rotation method was meted out by rotating the pictures in a horizontal direction with the most rotation angle of \pm 5 degrees, al-so, (iii) resized the CXR images to a hard and fast resized to a standard dimension, (iv) cropped to isolate the essential a part of the image and to delete certain orthogonal background objects, even to attenuate the commonly-found matter detail within the CXR images, (v) histogram adjustment technique was accustomed improve the distinction of every image, additionally to enhance the lighting conditions and therefore improving the training model. As seen in Fig. 3 as seen within the section on experimental outcomes. The pre-processed images are then divided into the training and testing assortment to evaluate the classification models.

$$
M(x, y)= \begin{cases}\max _{t h}, & i(x, y) \geq \min _{t h} \\ 0, & \text { otherwise }\end{cases}
$$

where $i(x, y)$ represents the input image, $\max _{t h}$ and $\min _{t h}$ are maximum and minimum thresholds mask design.

\subsection{Feature Extraction}

Extraction of options performs any transformation of the initial features by permitting variations and transformations of the original features set to develop new, additional important features to enhance the exactness of learning models by extracting features from input data. This method decreases the spatial property of the info by eliminating the redundant data and, thus, by protective the foremost wonderful elements, improves the speed and training. Extraction of feature is commonly used for describing the creation of the covariance matrix of incessant features with respectable discriminatory power between classes [14]. The salient and efficient features were extracted using nine simple morphic features (MFs) in 


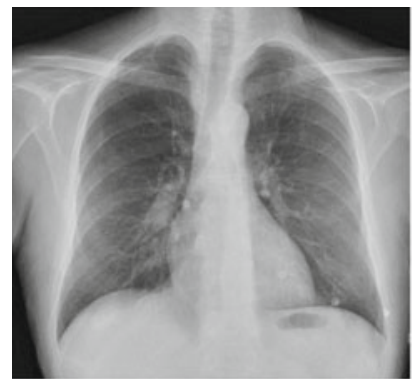

(a)

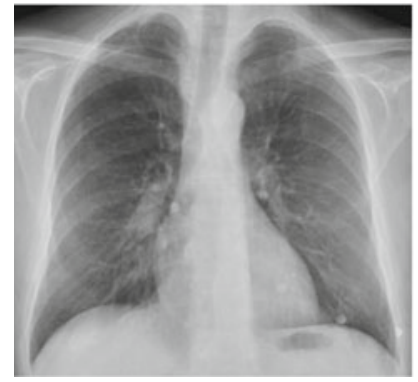

(c)

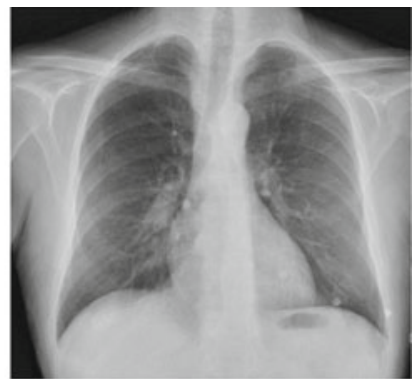

(b)

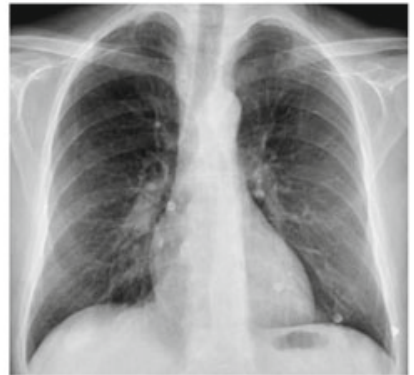

(d)

Fig. 3 Operation in data preprocessing: a original, $\mathbf{b}$ resized, $\mathbf{c}$ cropped, and $\mathbf{d}$ contrast-enhanced CXR image

addition to a collection of main components (PCs) were collected for the chosen X-ray image database, 4, 8, 16 and 24 PCs. PCs were derived from the intensive training collection. Namely, a given each image was interpreted as a row vector and its coefficients were then projected into a set of features; a new re-statement of the COVID-19 detail.

\section{Principal Component Analysis (PCA)}

Principal component analysis (PCA) may be a second-order applied mathematics approach that turns a group of related factors to lower range of non-correlated factors known as PCs. PCA is primarily accustomed cut back the size of the information set whereas maintaining the maximum amount details as possible rather than all the variance matrix PCs, the data are often expressed in mere a couple of straightforward vectors [15]. Computers were extracted from the complete training kit, where each image was presented as a carrier of a chain. Several characteristics have reduced the coefficients of each image; a new definition of the COVID-19 detail.

2. Morphological Features (MFs)

Morphological characteristics are derived from the apparent visual characteristics of a given image: (i) area: real number of pixels in the region; (ii) bounding box: represents the smallest measure box that contain all points; (iii) centriod: 
reflects the intersection of all straight lines separating a given image into two sections of the same moment of the line informally; (iv) convex area: represents a line segment that is completely enclosed within the form of any two points; (v) orientation: represents regions that can be defined as locally 1D (e.g. using lines or edges); and (vi) extreme: represents the largest value (maximum) or the smallest value (minimum) that takes place at a point, either within a neighbourhood or in a on the function domain in its entirety [16].

\subsection{Neutrosophic Techniques (NTS)}

After extracting the features using MFs and PCA, we employed the Neutrosophic techniques to extract three components: (i) membership degree (or T), (ii) indeterminacy degree (or I) and (iii) non-membership degree (or F). Those three components are correlated with the variables and are of immense importance for our classification algorithm in order to get more accurate and efficient diagnostic result.

Neutrosophic technique (NT) is a technique that would use the Neutrosophic logic rules and the Neutrosophic sets for grouping. Particularly, NT integrates a Neutrosophic rule-based method (e.g., IF X and Y THEN Z) for problem-solving instead of mathematically modeling the underlying system, i.e., NT is equivalent to a fuzzy technique [17]. The architecture of a Neutrosophic classification inference scheme using fuzzy terminology utilizes the Mamdani fuzzy inference method [18]. The block diagram of the Neutrosophic Classification Scheme is shown in Fig. 4. The membership functions ( $\mathrm{T}, \mathrm{I}$, and F) are mutually independent. Thus, using the Matlab fuzzy toolbox, three modules have been constructed: one for each of the Neutrosophic reality variable, Indeterminacy component, and the falsity component. Although independently for each other's components, a connection is drawn among the T, I, and F components to capture the truthfulness, in-determinacy and falseness of the input and the output [19].

In Neutrosophic-based classification (i.e., NRCS), rule-based systems using Neutrosophic logic are used to describe various types of information about the underlying topic and for modeling the connections and relationships that occur between its variables [20]. The generic structure of the NRCS is seen in Fig. 5.

Suppose U represents a discourse world that contains a subset $\mathrm{W}$ compromising bright pixels. Neutrosophical pictures are distinguished by three subsets T, I, and F. In the image, the pixel $\mathrm{P}$ in the image is defined as $\mathrm{P}(\mathrm{T}, \mathrm{I}, \mathrm{F})$ that relates to $\mathrm{W}$ by its $\mathrm{t} \%$ is true in the bright pixel, $\mathrm{i} \%$ is undetermined, and $\mathrm{f} \%$ is false. Here, $\mathrm{t}, \mathrm{i}$, and $\mathrm{f}$ vary in T, I, and F, respectively. Mathematically, a given pixel p (x, y) in the image domain, is converted to:

$$
N D P_{N s}(x, y)=\{T(x, y), I(x, y), F(x, y)\}
$$




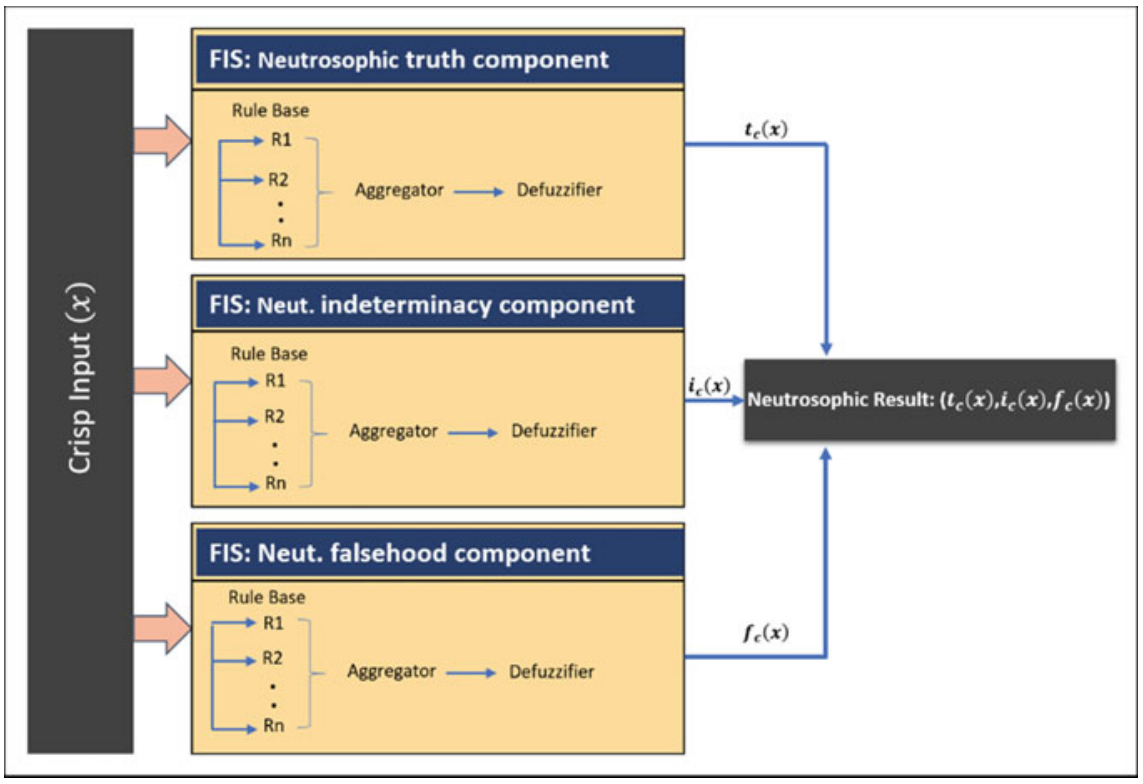

Fig. 4 Block diagram for Neutrosophic components

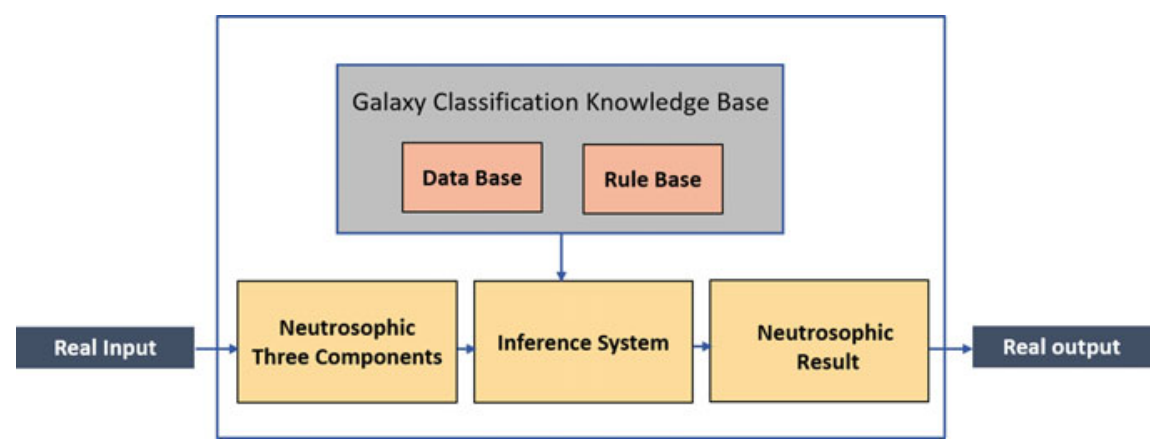

Fig. 5 The basic structure of a Neutrosophic rule-based classifier system

It refers to a white set, it applies to an indeterminate class and refers to a non-white set. Which could be described as [21]:

$$
\begin{gathered}
P_{N S}(x, y)=\{T(x, y), I(x, y), F(x, y)\} \\
T(x, y)=\frac{\overline{g(x, y)}-\bar{g}_{\text {min }}}{\bar{g}_{\text {max }}-\bar{g}_{\text {min }}}
\end{gathered}
$$




$$
\begin{gathered}
I(x, y)=1-\frac{H_{o}(x, y)-H_{o}}{H_{O_{\max }}-H_{o_{\min }}} \\
F(x, y)=1-T(x, y) \\
H_{o}(x, y)=a b s(g(x, y))-\overline{g(x, y)}
\end{gathered}
$$

where $\mathrm{g}(x, y)$ signifies the local mean value of the window size pixels, and Ho $(x, y)$ that could be distinct as the uniformity value of $\mathrm{T}$ at $(x, y)$ represented by the absolute difference between the intensity $\mathrm{g}(\mathrm{i}, \mathrm{j})$ and the local mean value $\mathrm{g}(x, y)$.

\subsection{Machine Learning and Classification}

Classification is often a part of the Diagnosis (CAD) system and plays a significant role in the medical imaging diagnosis [22]. In the final step of the proposed algorithm; we have used a multilayer perceptron (MLP) based classifier to make a learning process from the extracted features. After extracting the three components (membership, indeterminacy and non-membership) for each MFs and PCs features using NTs, the most robust component of the three components of every MFs and PCs namely membership component were fed to MLP classifier to categorize all the image into one of two cases: positive COVID-19 (+ve) or normal case negative COVID-19 (-ve).

MLP is front-feeding networks of layers that are typically trained on fixed back spread. These networks have made their way into a multitude of applications involving the Classification of set patterns. Its key advantage is that it's simple to use and round every internal/output diagram [23]. The multi-layer neural network consists of the input layer, the output layer, and more than a hidden layer capable of generating more teamwork effects. The key benefit is that it can reflect any logical and continuous function as long as the hidden units are appropriate and an acceptable activation function is used. The method of updating neural weights to accomplish goals is called positional propagation. It attempts in such a manner that it generates the least number of errors. Error is only apparent on the output layer, and this error is returned to the previous layers. The mistake is proportional to the combination of layers on the neural network. Finally, the new weights are revised and replicated again. Since the error size is high in the output layer, the same amount (proportion) of error is propagated back to the previous layer [24]. 


\section{Experimental Results and Discussion}

\subsection{Data Set Collection}

In this chapter, images of chest X-rays were utilized since this reading of radiography is often utilized by radiologists in clinical diagnosis. We tend to use a mix of two separate databases to construct our evaluation set. COVID-19 databases have been collected from in public access and picked up databases, whereas usual, and pneumonia databases have been compiled from publicly out there Kaggle databases:

- COVID-19 Dataset: A public database in GitHub have been built [25] by aggregating 319 X-rays of COVID-19, Middle East Respiratory Syndrome (MERS), Extreme Acute metabolism Syndrome (SARS) and respiratory disease from online resources and revealed articles. This database includes 250 positive X-rays of COVID-19 and 25 COVID-19 positive X-rays of lung CT images with a different image resolution. The COVID-19 database was extracted from this database to construct a new database.

- Normal (or Healthy) Subjects Database: The Kaggle Chest X-ray info may be an extremely popular database containing 5247 normal, infective agent and microorganism pneumonic x-rays of the chest with preciseness starting from $400 p$ to 2000p [26]. Of the 5247 chest X-rays, 3906 are from separate subjects with pneumonia (2561 footage of bacterial pneumonia and 1345 pictures of virus infection) and 1341 pictures of standard people. In this database, chest $\mathrm{x}$-rays of traditional and viral infection is employed to create an entire new database.

Table 1 shows the number of training and testing images for each type of chest X-ray. Figure 6 shows a sample of images taken from the database of X-ray images of the pulmonary chest COVID-19.

As shown in Fig. 7, X-rays are chest images taken from various patients with positive COVID-19 virus, and experienced radiologists examined the imaging results over a span of 20 years. Radiological findings are classified concerning [27] (i) peripheral predominance; a 72-year-old man complains of fever and cough. Initial chest $\mathrm{x}$-rays revealed a two-terminal opacity in the central and lower oceanic airspace regions (yellow arrows). The degree of severity was 2 per lung, therefore TSS was four (Fig. 7a), (ii) perihilar predominance, and 35-year-old males complained of coughing and fever. Primary chest X-ray revealed opacity to consolidate real oceanic air (yellow arrow). The darkness of the substitute lower-left airspace

Table 1 Summary of the training and testing data used for the evaluation of the proposed framework

\begin{tabular}{l|l|l|l}
\hline Type & Data set & Training set & Testing set \\
\hline COVID-19 subjects & 250 & 140 & 110 \\
\hline Normal subjects & 320 & 170 & 150 \\
\hline
\end{tabular}




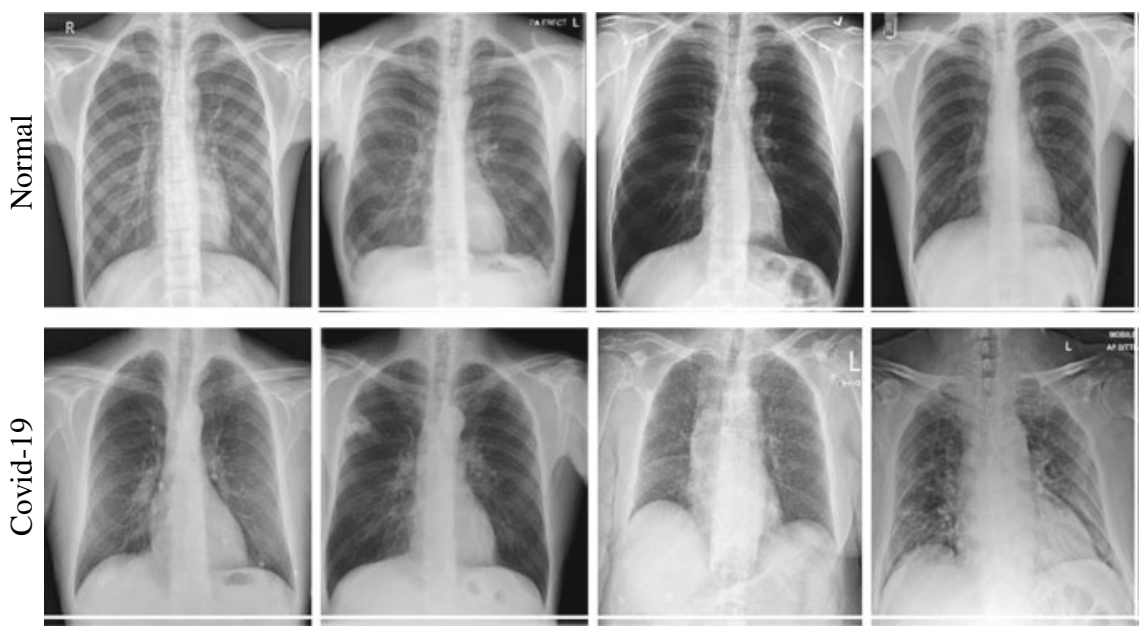

Fig. 6 Samples of chest X-ray images of two different cases: normal healthy person (first row), and patient suffering from covid-19 (second row)

(long shares) with internal depth was observed; the level of strength was the same for each lung, so TSS was a pair of (Fig. 7b), (iii) or neither; 27-year-old male whiney of cough and fever. Initial chest X-rays revealed minor pulmonary nodules in the center of the right area (yellow arrow) and neither peripheral nor oceanic space consolidation opacity (yellow arrows) was found, and the intensity score was 1 per lung, so TSS 2 (Fig. 7c), (iv) left, left, or binary lung and up; male 23 years without symptoms. Initial chest $\mathrm{x}$-rays revealed an opacity to improve the upper right area (yellow arrow).

The total severity was one (Fig. 7d), (v) and therefore the lower region; the 32-year male patient was whining of SOB and cough. Initial chest X-rays show the air space's blackout (yellow arrow) within the left and lower respiratory organ areas (with a lower area prevailing). The degree of intensity was 2 for the correct lung and a couple of on the left lung, therefore TSS four (Fig. 7e), (vi) the impact of the lung on monotheism, and the ground glass opacity (GGO); A 29-year-old feminine was affected by coughing and allergies. Initial chest x-rays showed a two-way glass opacity (yellow arrows) with a tiny low opacity within the higher left region (yellow arrow). The density was 2 per lung, so the TSS was 4 (Fig. 7f) (vii) was the reconfigured cellular thickness; the 48-year-old chest X-ray revealed the thickness of the binary biblical lung with opacity was still seen (yellow arrows) (Fig. 7g), (viii) and pulmonary nodules; the 49-year male was complaining of fever. Initial chest X-rays revealed two well-defined small pulmonary nodules in the middle lung region (yellow arrows). The degree of severity was 1 per lung, so TSS was 2 (Fig. 7h), (ix) had pleural or chest rest; the 61-year-old male complained of fever, cough, and diarrhea. Post-chest X-rays revealed a light left pleural effusion with a bilateral opacity to help the lower logical air region (yellow arrows). 


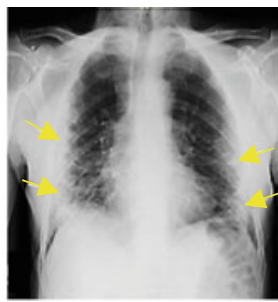

(a)

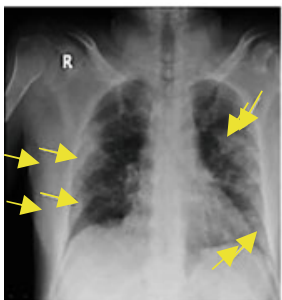

(e)

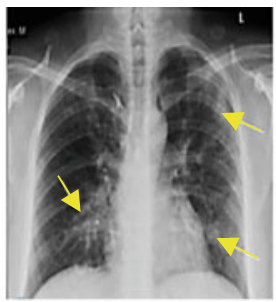

(b)

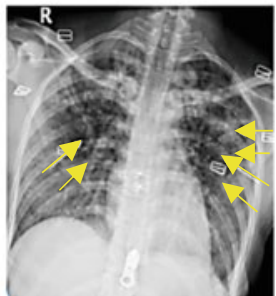

(f)

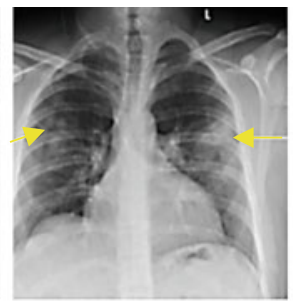

(c)

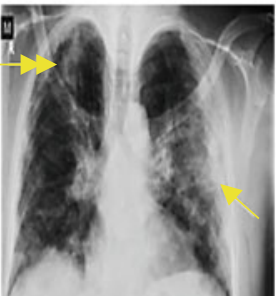

(g)

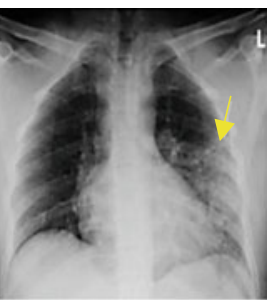

(d)

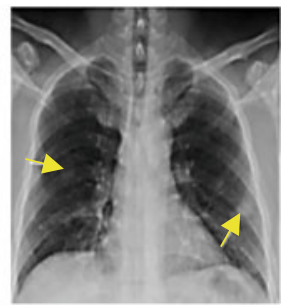

(h)

Fig. 7 Examples of chest X-rays for different patients with positive COVID-19

\subsection{Performance Measures}

In order to assess the potency of the planned algorithm, varied strategies are accustomed to determine the afflicted person $(+v e)$ and, therefore, the normal person (-ve) within the X-rays images studied. The cross-checkable was used and resulted in an uncertainty matrix, as seen in Fig. 8. The confusion matrix consists of 4 predicted findings are as follows.

Four main components are used to generate the confusion matrix True positive (TP); True negative (TN), False positive (FP), and False negatives (FN). TP may be an assortment of abnormalities that are detected with the correct diagnosis. TN is an incorrectly calculated variety of traditional ideals. FP is a set of normal cases referred to as FP for the detection of abnormalities. Finally, FN is a list of anomalies

\begin{tabular}{|c|c|c|c|}
\hline & \multicolumn{2}{|c|}{$\begin{array}{c}\text { Actual Value } \\
\text { (as confirmed bv experiment) }\end{array}$} \\
\hline & & Positive & Negative \\
\hline \multirow{2}{*}{ 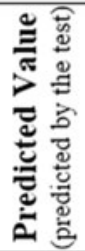 } & Positive & True Positive (TP) & False Positive (FP) \\
\hline & Negative & False Negative (FN) & True Negative (TN) \\
\hline
\end{tabular}

Fig. 8 Confusion matrix 
that have been determined as an usual diagnosis. Quality metrics may be determined by measure the values of the doable outcomes within the confusion matrix, Output metrics are often measured. Check trained models are analyzed exploitation common output metrics like precision, accuracy(ACC), recall (selective), specificity, and F1 score (dice coefficient). It shall be represented as follows:

\section{Accuracy}

It is a parameter that tests the method's capacity by accurately calculating the proportion of cases predicted from all cases. ACC is mathematically expressed as follows [28]:

$$
A C C=\frac{T P+T N}{T P+F P+F N+T N}
$$

where TP (TN) is the correctly predicted positive (negative) cases and FP (FN) is the incorrectly predicted positive (negative) cases. However, exactness isn't invariably smart for evaluating every model type, notably within the case of an associate uneven knowledge set. there's conjointly a requirement to research alternative success metrics to validate the model.

\section{Precision}

Precision represents the magnitude relation of well-anticipated positive cases to the general expected positive cases. High accuracy compares to the poor, inaccurate positive rate. It's expressed as [29]:

$$
\text { Precision }=\frac{T P}{T P+F P}
$$

\section{Recall (Sensitivity)}

This is the magnitude relation of the properly expected positive notes to any or all operations within the actual class i.e. true positive rate. The confusion matrix is employed to assess sensitivity and is mathematically assessed as [30]:

$$
\text { Recall }(\text { Sensitivity })=\frac{T P}{T P+F N}
$$

\section{Specificity}

It is that the proportion of accurately forecast negative notes on any or more of the real negative observations [31]:

$$
\text { Specificity }=\frac{T N}{F P+T N}
$$




\section{F1-Score}

The F1-score may be a comprehensive calculation of the accuracy of the model that blends exactness and recollection, the F1-score is double the quantitative relation between the multiplication of accuracy and also the retrieval measurement. Provides a balance between precision and recall [29]:

$$
F 1-\text { Score }=\frac{2 \times \text { Precision } \times \text { Recall }}{\text { Precision }+ \text { Recall }}
$$

\subsection{Results and Discussion}

The prompt design has been trained using the following criteria: two hidden layers, and 1000 epochs. The suggested framework has been applied employing a software package kit (MATLAB 2017b). Computer-implementation was for CPU and works during a 64-bit Windows environment. Each tests were conducted on the Intel $\mathrm{R}$ processor $(2.60 \mathrm{GHz})$ and $4.00 \mathrm{~GB}$ Ram. Five collections of options are arranged: (i) morphological features only; (ii) morphological features of 4 PCs; (iii) Morphological options with eight computers; (iv) morphological features with sixteen computers; and (v) morphological features with twenty four computers. There is no reason to extract extra machines due to the information indicating no clear distinction within the utilization of over 24 PCs. Then, we tend to used NTs techniques together with the MLP classifier. NTs are additional to the three Neutrosophic parts' derived options, i.e. participation, non-identification and in organism, the foremost potent element of the three components per radiofrequency. Therefore, the machine is that the organic component that was fed to the MLP classifier. Figure 9 displays a graph of the Neutrosophic Components (NCs) graph; a sample of all MFs with machine features in the Neutrosophic environment.

The proposed algorithm's performances were measured by employing various performance metrics - confusion matrix, accuracy, precision; sensitivity, specificity, and F1-Score. Figure 10 shows the fusion matrix summary of the proposed algorithm, TP is that true positive within the case of COVID-19, and TN is the true negative in the normal state. In contrast, FP and FN are wrong model estimates for COVID-19 and also the alternative case.

Although the results are seen in Table 2 and Fig. 11 it's been found that the MLP-based compiler of MFs with options of 24 PCs features the most effective results while not exploiting NTs for all the cases tested for geared up of distinctive features, but once applied NTs to any or all cases tested for all set of features, we tend to found the results showing this using NTs of (MFs with 4PCs) in combination with MLP based classifier provides better result than using the MLP classifier with the other groups of features: $\mathrm{ACC}=0.9846$; precision $=0.9818$; sensitivity $=0.9818$, specificity $=0.9867$ and F1-Score $=0.9818$. 


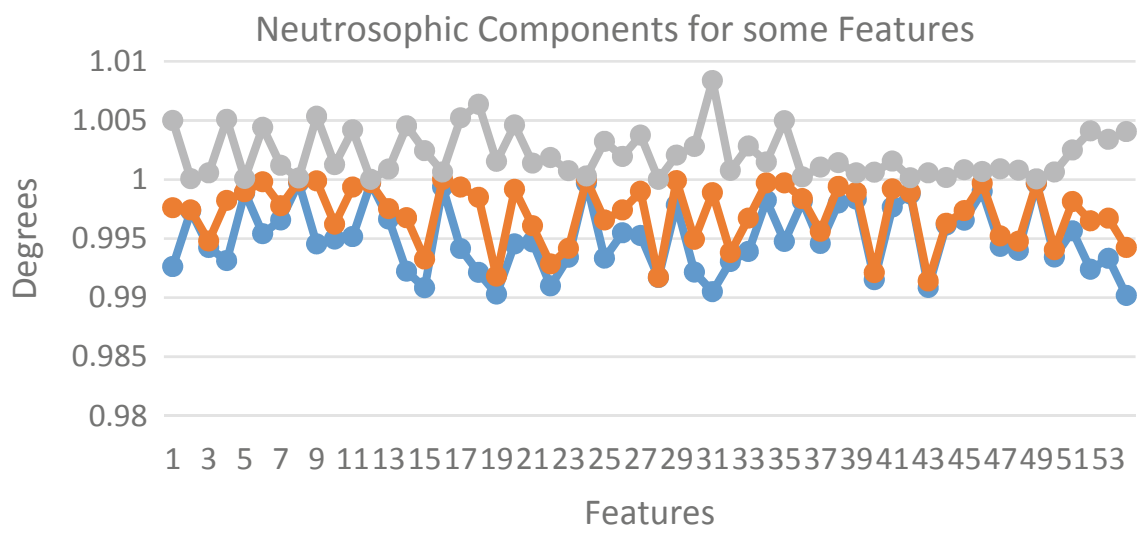

$\longrightarrow$ Membership (T) Indeterminacy (I) $\longrightarrow$ Non-membership (F)

Fig. 9 Neutrosophic three components for some features

\begin{tabular}{|c|c|c|c|}
\hline & \multicolumn{2}{|c|}{ Actual Covid-19 } \\
\hline & & (+ve) & $(-v e)$ \\
\hline \multirow{2}{*}{ 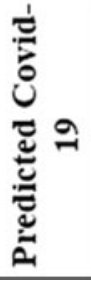 } & (+ve) & $\begin{array}{c}\text { True Positive } \\
108\end{array}$ & $\begin{array}{c}\text { False Positive } \\
2\end{array}$ \\
\hline & (-ve) & $\begin{array}{c}\text { False Negative } \\
2\end{array}$ & $\begin{array}{c}\text { True Negative } \\
148\end{array}$ \\
\hline
\end{tabular}

Fig. 10 Confusion matrix analysis of the NCs for (MFs + 4 Pcs)

This means that the algorithm relies on a small number of tested features for Classification, i.e. only MFs with 4PCs, which saves time and reduces system complexity while achieving higher efficiency in the classification process. This means, that a small set of features is sufficient to classify COVID-19 images using the NTs also there no any need for much more than 4 PCs with using NTs since the results show no noticeable difference.

Table 3 represents a comparison result between the proposed architecture and the other related works which indicate that our approach has worked better for classifying the images of chest X-ray into two classes. Also achieved a high-performance, efficiency, simplicity, and speedy in Classification. 
Table 2 Performance of the proposed framework

\begin{tabular}{l|l|l|l|l|l|l}
\hline $\begin{array}{l}\text { Performance } \\
\text { measures }\end{array}$ & MFs & $\begin{array}{l}\text { MFs + 4 } \\
\text { PCs }\end{array}$ & $\begin{array}{l}\text { MFs + 8 } \\
\text { PCs }\end{array}$ & $\begin{array}{l}\text { MFs + 16 } \\
\text { PCs }\end{array}$ & $\begin{array}{l}\text { MFs + 24 } \\
\text { PCs }\end{array}$ & $\begin{array}{l}\text { NT for } \\
\text { MFs + 4 PCs }\end{array}$ \\
\hline Accuracy & 0.9038 & 0.9462 & 0.9577 & 0.9692 & 0.9731 & 0.9846 \\
\hline Precision & 0.8636 & 0.9091 & 0.9364 & 0.9545 & 0.9636 & 0.9818 \\
\hline Sensitivity & 0.9048 & 0.9615 & 0.9626 & 0.9722 & 0.9725 & 0.9818 \\
\hline Specificity & 0.9032 & 0.9359 & 0.9542 & 0.9671 & 0.9735 & 0.9867 \\
\hline F1-Score & 0.8837 & 0.9346 & 0.9493 & 0.9633 & 0.9680 & 0.9818 \\
\hline
\end{tabular}

Note that "MF", "PC" and "NC" stand for morphological features, principal components, and Neutrosophic technique, respectively

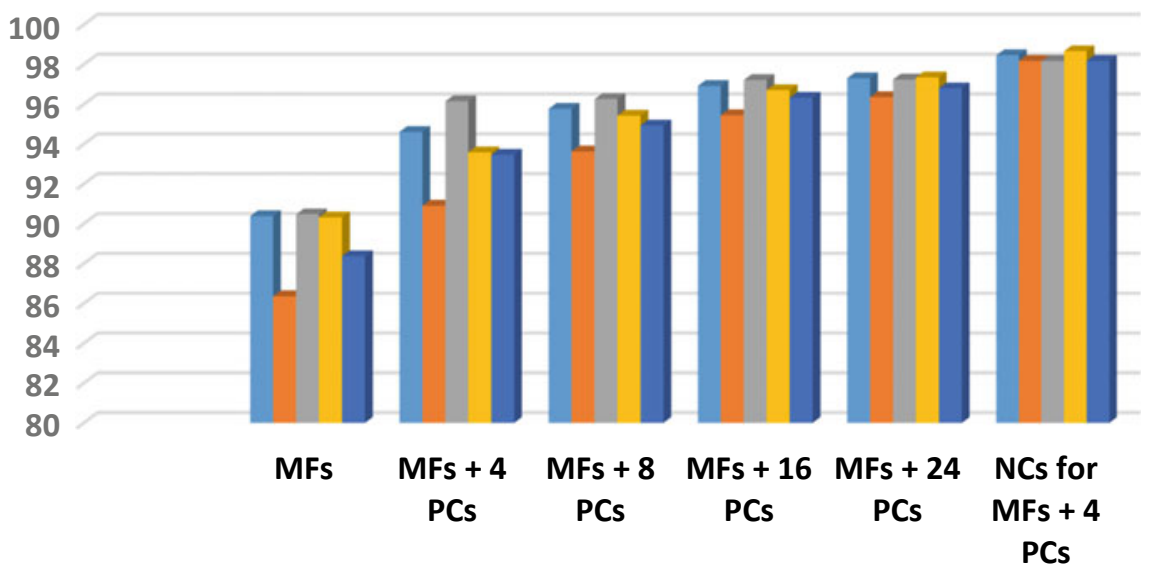

\section{Accuracy \% $\quad$ Precision \% $\quad$ Sensitivity \% $\quad$ Specificity \% $\quad$ F1-Score \%}

Fig. 11 The performance metrics of the proposed pipeline for COVID-19 diagnosis

Table 3 The proposed architecture results and the other related works

\begin{tabular}{l|l|l|l|l|l}
\hline Performance measures (\%) & Accuracy & Precision & Sensitivity & Specificity & F1-Score \\
\hline $\begin{array}{l}\text { Apostolopoulos and Mpesiana } \\
\text { [7] }\end{array}$ & 96.78 & NA & 98.66 & 96.46 & NA \\
\hline Sethy and Behera [8] & 95.38 & NA & NA & NA & 95.52 \\
\hline Afshar et al. [10] & 95.7 & NA & 90 & 95.8 & NA \\
\hline Abbas et al. [12] & 95.12 & NA & 97.91 & 91.87 & NA \\
\hline Proposed & $\mathbf{9 8 . 4 6}$ & $\mathbf{9 8 . 1 8}$ & $\mathbf{9 8 . 1 8}$ & $\mathbf{9 8 . 6 7}$ & $\mathbf{9 8 . 1 8}$ \\
\hline
\end{tabular}




\section{Conclusion}

This paper has proposed a novel, robust, automated, and intelligent system for COVID-19 Classification using chest X-rays. The proposed pipeline is a hybrid technique that integrates machine learning techniques and Neutrosophic techniques. The testing accuracy, precision, sensitivity, specificity, and F1-Score for the scheme were $98.46 \%, 98.19 \%, 98.18 \%$, and $98.67 \%, 98.17 \%$. The obtained results show that using MFs with 4PCs for feature extraction in combination with Neutrosophic techniques and multi-layer perceptron-based classifier provides the best results compared to other ways. This will be significantly helpful during this natural event once the sickness and the want for hindrance action are in distinction with available resources. Our proposed pipeline's weakness is that it only offers a straightforward positive or negative COVID-19 observation to the physician. It will act as a screening aid to a medical imaging specialist. Our future research will be dedicated to providing staging for the severity of COVID-19 cases. This will be achieved by using more possible multi-database algorithms with clinical information for multi-modelling for improved prediction of the disease and deployment of the application in the real world.

\section{References}

1. Chowdhury, M.E., Rahman, T., Khandakar, A., Mazhar, R., Kadir, M.A., Mahbub, Z.B., Islam, K.R., Khan, M.S., Iqbal, A., Al-Emadi, N., Reaz, M.B.I.: Can AI help in screening viral and COVID-19 pneumonia? (2020) arXiv preprint arXiv:2003.13145

2. Punn, N.S., Agarwal, S.: Automated diagnosis of COVID-19 with limited posteroanterior chest X-ray images using fine-tuned deep neural networks (2020). arXiv preprint arXiv:2004.11676

3. Wang, W., Xu, Y., Gao, R., Lu, R., Han, K., Wu, G., et al.: Detection of SARS-CoV-2 in different types of clinical specimens. Jama (2020)

4. Yang, T., Wang, Y.-C., Shen, C.-F., Cheng, C.-M.: Point-of-care RNA-based diagnostic device for COVID-19. Multidisciplinary Digital Publishing Institute (2020)

5. Huang, C., Wang, Y., Li, X., Ren, L., Zhao, J., Hu, Y., et al.: Clinical features of patients infected with 2019 novel coronavirus in Wuhan, China. Lancet 395, 497-506 (2020)

6. Allen, J.N., Davis, W.B.: Eosinophilic lung diseases. Am. J. Respir. Crit. Care Med. 150(5), 1423-1438 (1994)

7. Apostolopoulos, I.D., Mpesiana, T.A.: Covid-19: automatic detection from x-ray images utilizing transfer learning with convolutional neural networks. Phys. Eng. Sci. Med. 1, 635640 (2020). https://doi.org/10.1007/s13246-020-00865-4

8. Sethy, P.K., Behera, S.K.: Detection of coronavirus disease (covid-19) based on deep features. Preprints 2020030300, 2020 (2020)

9. Khalifa, N.E.M., Taha, M.H.N., Hassanien, A.E., Elghamrawy, S.: Detection of coronavirus (covid-19) associated pneumonia based on generative adversarial networks and a fine-tuned deep transfer learning model using chest x-ray dataset (2020). arXiv:2004.01184

10. Afshar, P., Heidarian, S., Naderkhani, F., Oikonomou, A., Plataniotis, K.N., Mohammadi, A.: Covid-caps: a capsule network-based framework for identification of covid-19 cases from x-ray images (2020). arXiv preprint arXiv:2004.02696 
11. Wang, L., Lin, Z.Q., Wong, A.: Covid-net: a tailored deep convolutional neural network design for detection of covid-19 cases from chest x-ray images. Sci. Rep. 10(1), 1-12 (2020)

12. Abbas, A., Abdelsamea, M.M., Gaber, M.M.: Classification of COVID-19 in chest X-ray images using DeTraC deep convolutional neural network (2020). arXiv preprint arXiv:2003.13815

13. OpenCV: Image thresholding (2020). https://docs.opencv.org/master/d7/d4d/tutorialpythresholding. html. Online; Accessed 12 April 2020

14. Khalid, S., Khalil, T., Nasreen, S.: A survey of feature selection and feature extraction techniques in machine learning. In: 2014 Science and Information Conference, pp. 372-378. IEEE (2014)

15. Ruck, D., Rogers, S., Kabrishy, M.: Feature selection using a multilayer perceptron. J. Neural Netw. Comput. 2(2), 40-48 (1990)

16. Jing, X.J., Yu, N., Shang, Y.: Image filtering based on mathematical morphology and visual perception principle. Chin. J. Electron. 13.4, 612-616 (2004)

17. Ansari, A.Q., Biswas, R., Aggarwal, S.: Neutrosophic classifier: an extension of fuzzy classifer. Appl. Soft Comput. 13(1), 563-573 (2013)

18. Mohammed, M.N., Syamsudin, H., Al-Zubaidi, S., AKS, R.R., Yusuf, E.: Novel COVID-19 detection and diagnosis system using IOT based smart helmet. Int. J. Psychosoc. Rehabil. 24 (7), 2296-2303 (2020)

19. Yasser, I., Twakol, A., El-Khalek, A., Samrah, A., Salama, A.A.: COVID-X: novel health-fog framework based on neutrosophic classifier for confrontation covid-19. Neutrosophic Sets Syst. 35(1), 1 (2020)

20. Fang, Y., Zhang, H., Xie, J., Lin, M., Ying, L., Pang, P., Ji, W.: Sensitivity of chest CT for COVID-19: comparison to RT-PCR. Radiology 200432 (2020)

21. Salama, A.A., Smarandache, F., Eisa, M.: Introduction to image processing via neutrosophic techniques. Neutrosophic Sets Syst. 5, 59-64 (2014)

22. Dey, N.: Classification Techniques for Medical Image Analysis and Computer Aided Diagnosis. Academic Press (2019)

23. Mohammadian, A., Miller, E.J.: Nested logit models and artificial neural networks for predicting household automobile choices: comparison of performance. Transp. Res. Rec. 1807(1), 92-100 (2002)

24. Biswas, M., Adlak, R.: Classification of galaxy morphologies using artificial neural network. In: 2018 4th International Conference for Convergence in Technology (I2CT), pp. 1-4. IEEE (2018)

25. Monteral, J.C.: COVID-Chestxray Database (2020). Available: https://github.com/ieee8023/ covid-chestxray-dataset

26. Mooney, P.: Chest X-Ray Images (Pneumonia) (2018). Available: https://www.kaggle.com/ paultimothymooney/chest-xray-pneumonia

27. Yasin, R., Gouda, W.: Chest X-ray findings monitoring COVID-19 disease course and severity. Egypt. J. Radiol. Nucl. Med. 51(1), 1-18 (2020)

28. Jain, G., Mittal, D., Thakur, D., Mittal, M.K.: A deep learning approach to detect covid-19 coronavirus with X-Ray images. Biocybernetics Biomed. Eng. 40(4), 1391-1405 (2020)

29. Hemdan, E.E.-D., Shouman, M.A., Karar, M.E.: Covidx-net: a framework of deep learning classifiers to diagnose covid-19 in X-ray images (2020). arXiv preprint arXiv:2003.11055

30. Pannu, H.S., Singh, D., Malhi, A.K.: Improved particle swarm optimization based adaptive neuro-fuzzy inference system for benzene detection. CLEAN-Soil Air Water 46(5), 1700162 (2018)

31. Kaur, M., Gianey, H.K., Singh, D., Sabharwal, M.: Multiobjective differential evolution based random forest for e-health applications. Mod. Phys. Lett. B 33(05), 1950022 (2019) 